\title{
Effect of Xinyue capsules on patients with coronary heart disease after percutaneous coronary intervention: study protocol for a randomized controlled trial
}

Ming Guo ${ }^{1}$, Ming-jie Zi ${ }^{1}$, Rui-xi Xi ${ }^{1,2}$, Qiao-ning Yang ${ }^{1}$, Rui-na Bai ${ }^{1}$, Yi-sheng Zhang ${ }^{2}$, Yu-hua Wang ${ }^{2}$, Pei-li Wang ${ }^{1 *}$ and Da-zhuo Shi ${ }^{1}$

\begin{abstract}
Background: The risk of cardiovascular events remains high in patients with coronary heart disease (CHD) after successful percutaneous coronary intervention (PCI). Panax quinquefolius saponin, a major component of Xinyue capsule, has been used to treat patients with CHD. The aim of this study is to evaluate the efficacy and safety of Xinyue capsules in patients with CHD after PCl.

Methods/design: This study is a multicenter, placebo-controlled, double-blind, randomized controlled clinical trial. A total of 1100 participants are randomly allocated to two groups: the intervention group and a placebo group. The intervention group receives Xinyue capsules plus conventional treatment, and the placebo group receives placebo capsules plus conventional treatment. The patients receive either Xinyue or placebo capsules three times daily $(1.8 \mathrm{~g} /$ day) for up to 24 weeks. The primary outcome measure is the time from randomization to the first occurrence of major adverse cardiovascular events. The secondary outcome measure is the time from randomization to the first occurrence of stroke, pulmonary embolism, and peripheral vascular events, as well as death due to any cause. All outcome measures will be assessed at 12, 24, 36, and 48 weeks after randomization. Adverse events will be monitored during the trial.
\end{abstract}

Discussion: The aim of this study is to evaluate the effects of Xinyue capsules on patients with CHD after interventional treatment. The results of this trial will provide critical evidence regarding Chinese herbal medicine treatment for $\mathrm{CHD}$.

Trial registration: Chinese Clinical Trials Registry identifier ChiCTR-IPR-14005475. Registered on 10 November 2014.

Keywords: Xinyue capsule, Coronary heart disease, Randomized controlled trial

\section{Background}

Percutaneous coronary intervention (PCI) is commonly performed for coronary revascularization in patients with stable angina or acute coronary syndrome (ACS) [1]. Nevertheless, there are several medications with proven benefit to patients with cardiovascular disease, such as dual-antiplatelet therapy, statins, $\beta$-blockers, and angiotensin-converting enzyme inhibitors [2]. Unfortunately,

\footnotetext{
* Correspondence: 191593690@qq.com

${ }^{1}$ Cardiovascular Diseases Center, Xiyuan Hospital, China Academy of Chinese Medical Sciences, 1 Xiyuan Caochang, Haidian District, Beijing 100091, China Full list of author information is available at the end of the article
}

the risk of cardiovascular events remains high in patients after PCI [3]. Many patients do not receive the conventional treatment due to the side effects, contraindications, and drug-drug interactions [4]. Selecting the optimal clinical strategies to prevent the occurrence of cardiovascular events is challenging.

Traditional Chinese medicine (TCM) has been used to treat coronary heart disease (CHD) for thousands of years [5]. From the perspective of TCM, patients with CHD can be divided into different syndromes (i.e., different zhengs). In the diagnosis of CHD, the "Qi and Yin inadequacy syndrome" is the important 
subtype. In 2005, Xinyue capsules were approved by the China Food and Drug Administration for treatment of CHD. The main component of Xinyue capsules-Panax quinquefolius saponin (PQS)-is extracted from the stem and leaves of Panax quinquefolium, which in TCM theory could reinforce Qi and nourish Yin. Previous studies have shown that PQS has various pharmacological actions, including antimyocardial cell damage [6], protection of heart function, reduction of myocardial oxygen consumption $[7,8]$, improvement of myocardial perfusion [9] and ventricular remodeling after acute myocardial infarction (MI) [10], antiapoptosis of ischemic myocardial cells [11, 12], regulation of glucose and lipid metabolism [13], and improvement of insulin resistance [14, 15]. A multicenter randomized clinical study [16] showed that the combination of Xinyue capsules with Chuanxiong capsules and conventional Western interventions could reduce the occurrence of cardiovascular events in patients with ACS after PCI without increasing the risk of major bleeding. The results of pharmaceutical chemistry and pharmacokinetics conducted jointly by Xiyuan Hospital and Medical University of Vienna showed that the fingerprint of Xinyue capsules from different batches or the same batch at different time points was consistent, and the measurements of heavy metal and pesticide residues were within European Union standards.

Based on the multifactorial effect on CHD of Xiyue capsule, our hypothesis is that Xiyue capsules plus conventional treatment can improve cardiovascular outcomes in patients with CHD after interventional treatment compared with conventional treatment alone. If successful, it will provide a novel, promising alternative strategy for further reducing cardiovascular events.

\section{Methods/design}

\section{Study design}

This study is registered in the Chinese Clinical Trials Registry (ChiCTR-IPR-14005475). It is a multicenter, double-blind, randomized, placebo-controlled clinical trial. This study complies with the principles of the Declaration of Helsinki and Good Clinical Practice guidelines. Written informed consent will be obtained from all patients prior to their participation in this study, and the recruited patients will be randomized to either the Xiyue capsule group or the placebo group. We will rigorously follow the Consolidated Standards of Reporting Trials (CONSORT) recommendations in reporting the results [17].

The trial will be conducted in 25 centers in China (see in Additional file 1). A total of 1100 participants will be recruited. After acquiring consent from the participants or their parents and/or legal guardians, the participants will be enrolled in the trial, which consists of a 1-week run-in period, a 24-week treatment period, and a 24week follow-up period. An outline of the study procedures is illustrated in Fig. 1.

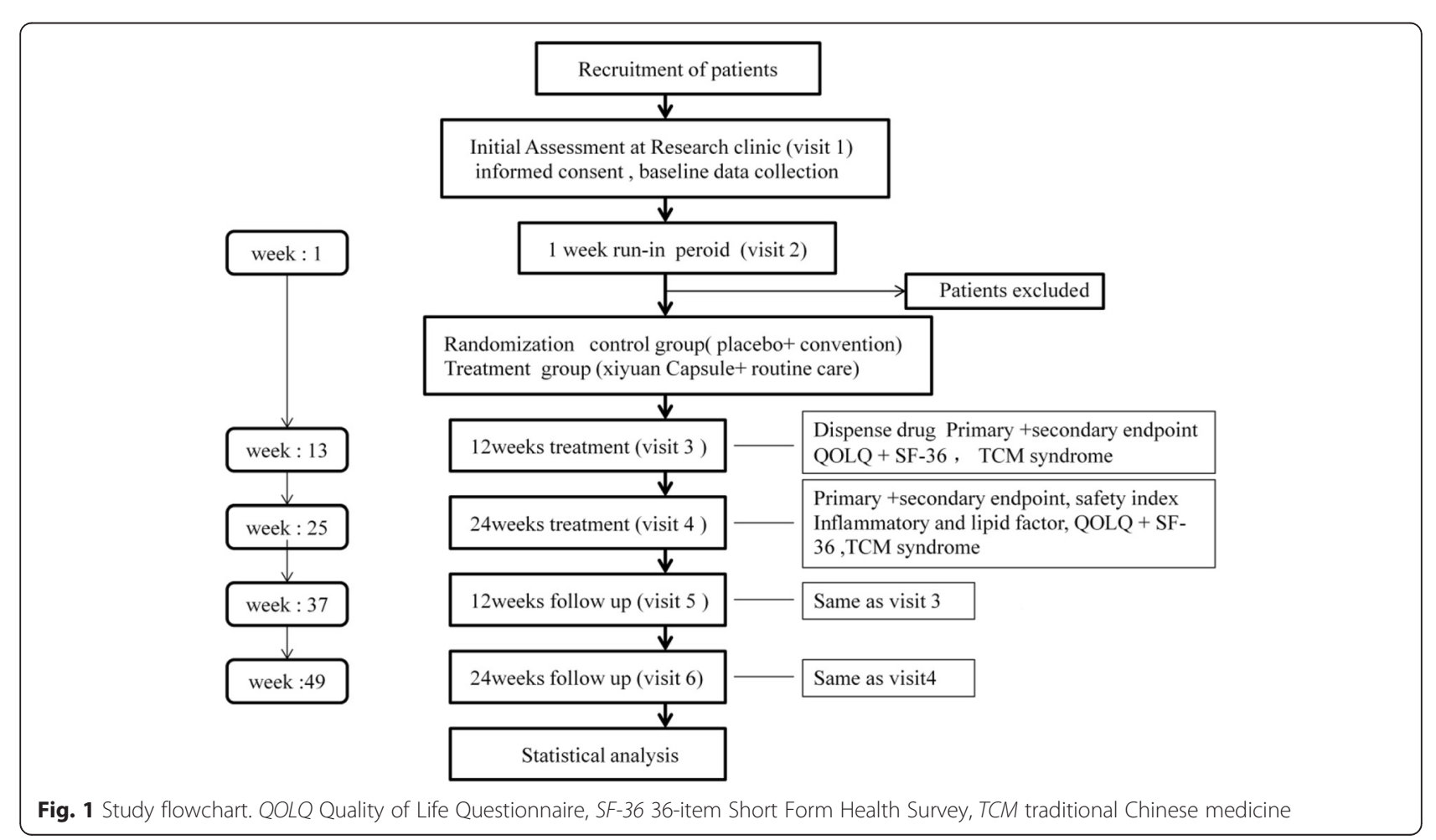




\section{Participants}

Inpatients and outpatients in the participating centers will be screened if they meet the selection criteria.

\section{Inclusion criteria}

1. CHD was diagnosed according to the guidelines $[2,18]$. All participants must have received successful PCI.

2. The time window is within 1 year after PCI and the patient must be clinically stable, defined as follows:

a. No progressive chest pain is present.

b. The troponin value is within normal range or slightly elevated, but lower than the 99th percentile of the upper reference limit value.

c. The participants must have a Canadian Cardiovascular Society classification of angina I to II

3. Patients are diagnosed as having a syndrome of deficiency of both Qi and Yin according to TCM standards [19].

4. Patients must be aged $18-75$ years.

5. Patients must have a New York Heart Association cardiac functional class I-II

6. Patients are informed about the trial and voluntarily sign the consent form.

\section{Exclusion criteria}

1. Renal insufficiency, male serum creatinine $>2.5 \mathrm{mg} /$ $\mathrm{dl}(>220 \mu \mathrm{mol} / \mathrm{L})$, female serum creatinine $>2.0 \mathrm{mg} /$ $\mathrm{dl}(>175 \mu \mathrm{mol} / \mathrm{L})$

2. Serious liver disease or alanine transaminase and aspartate transaminase values two times higher than the upper reference limit value

3. Systolic blood pressure $>160 \mathrm{mmHg}$ or diastolic blood pressure $>100 \mathrm{mmHg}$

4. Presence of diabetes with random blood glucose level $\geq 13.7 \mathrm{mmol} / \mathrm{L}$ or $\mathrm{HbA} 1 \mathrm{c} \geq 9.5 \%$

5. Pregnant women or women who are preparing for pregnancy, and subjects who are known to be allergic to ingredients of the study drug

6. Acute cerebrovascular disease

7. Malignancy or having a life expectancy less than 3 years

8. Severe hematopoietic diseases

9. Severe psychiatric conditions

10. Previous involvement in other clinical trials or participation in other clinical trials within the past 3 months

\section{Withdrawal, dropout, and discontinuation}

Participants can voluntarily withdraw at any time during the trial. Subjects who meet the inclusion criteria, fill out and sign the consent form, and successfully enter the randomized trial who fail to complete the observational period proposed in the trial, regardless of time and reasons, are considered as dropout cases. Reasons for withdrawal will be recorded in case report forms (CRFs), and the last data recorded for these participants will be included in the data analysis.

The trial can be terminated in the following circumstances: (1) occurrence of serious adverse events (AEs) related to the research medication, (2) the test drug is found to have no clinical value during the trial, (3) financial and management reasons, and/or (4) administrative authorities terminate the trial.

\section{Intervention}

Eligible patients will be allocated to receive Xinyue capsule or placebo for 6 months in addition to conventional treatment, including antiplatelet, lipid-lowering, antihypertensive, or antidiabetic therapy, according to the clinical guidelines. The Xinyue capsules and placebo capsules $(0.6 \mathrm{~g}$ ante cibum, three times daily for 24 weeks; China State Food and Drug Administration approval number Z20030073) will be provided by Jilin Jilin Jian Yisheng Pharmaceutical Co. Ltd. (Ji'An City, China). Each capsule is $0.3 \mathrm{~g}$, equivalent to $50 \mathrm{mg}$ of $P$. quinquefolium saponin. The Xinyue and placebo capsules have the same outer packaging, color, shape, and flavor.

\section{Outcomes and measures}

The primary outcome is the time from randomization to the first occurrence of the major adverse cardiovascular event (MACE): cardiac death (any death unless an unequivocal noncardiac cause could be established), nonfatal MI (appearance of pathological $\mathrm{Q}$ waves that were absent at baseline or a total creatine kinase [CK] level more than two times the upper limit of normal [ULN] with presence of CK isoenzyme MB higher than the ULN), and urgent revascularization with either PCI or coronary artery bypass graft. The secondary outcome measures is the time from randomization to the first occurrence of, stroke, pulmonary embolism, and peripheral vascular events, as well as death due to any cause. Safety outcomes, which include a complete blood count, kidney and liver function tests, high-sensitivity C-reactive protein level, cardiac function index, blood lipid levels, Quality of Life Questionnaire (QLQ) score, 36item Short Form Health Survey (SF-36) score, and TCM syndrome score will also be monitored periodically. All AEs will be followed from randomization to the end of the trial. Items to be measured and the time window of data collection are shown in Table 1. 
Table 1 Measurement items and points of data capture

\begin{tabular}{|c|c|c|c|c|c|c|}
\hline & Screening & Treatment peri & & & Follow-up & \\
\hline & Visit 1 & $\begin{array}{l}\text { Run-in period } \\
\text { (visit 2) }\end{array}$ & $\begin{array}{l}12 \text { weeks } \\
\text { (visit 3) }\end{array}$ & $\begin{array}{l}24 \text { weeks } \\
\text { (visit 4) }\end{array}$ & $\begin{array}{l}12 \text { weeks } \\
\text { (visit 5) }\end{array}$ & $\begin{array}{l}24 \text { weeks } \\
\text { (visit 6) }\end{array}$ \\
\hline Informed consent & $x$ & & & & & \\
\hline Inclusion/exclusion criteria & & $x$ & & & & \\
\hline Demographic data & $x$ & & & & & \\
\hline Medical history & $x$ & & & & & \\
\hline Concomitant medications & $x$ & & & & & \\
\hline Tongue and pulse condition in TCM & $x$ & $x$ & $x$ & $x$ & $x$ & $x$ \\
\hline Vital signs & $x$ & $x$ & $x$ & $x$ & $x$ & $x$ \\
\hline 12-lead ECG & $x$ & & & $x$ & & $x$ \\
\hline $\begin{array}{l}\text { Complete blood count, urine } \\
\text { and stool tests }\end{array}$ & $x$ & & & $x$ & & $x$ \\
\hline Liver and renal function tests & $x$ & & & $x$ & & $x$ \\
\hline Coagulation function test & $x$ & & & $x$ & & $x$ \\
\hline Adverse event evaluation & & & $x$ & $x$ & $x$ & $x$ \\
\hline Dispense drug & & $x$ & $x$ & $x$ & $x$ & $x$ \\
\hline Primary endpoint & & & $x$ & $x$ & $x$ & $x$ \\
\hline Secondary endpoint & & & $x$ & $x$ & $x$ & $x$ \\
\hline Cardiac function & $x$ & & & $x$ & & $x$ \\
\hline hs-CRP & $x$ & & & $x$ & & $x$ \\
\hline Lipid panels & $x$ & & & $x$ & & $x$ \\
\hline QLR score & $x$ & & $x$ & $x$ & $x$ & $x$ \\
\hline SF-36 score & $x$ & & $x$ & $x$ & $x$ & $x$ \\
\hline TCM syndrome score & $x$ & & $x$ & $x$ & $x$ & $x$ \\
\hline
\end{tabular}

Abbreviations: ECG electrocardiogram, hs-CRP high-sensitivity C-reactive protein, SF36 36-item Short Form Health Survey, TCM traditional Chinese medicine, QLQ quality of Life Questionnaire

$X$ represents the indicators tested in the specific time period

Coagulation function test: prothrombin time, activated partial thromboplastin time, fibrinogen, thrombin time

Lipid panels: high-density lipoprotein, low-density lipoprotein, cholesterol, triglycerides

Primary endpoint: time from randomization to the first occurrence of the major adverse cardiovascular event

Secondary endpoint: time from randomization to the first occurrence of stroke, pulmonary embolism, and peripheral vascular events, as well as death due to any cause

\section{Adverse events}

AEs are defined as negative or unintended clinical manifestations following the treatment. Patients will be asked to report to the investigators any abnormal reactions occurring at any time during the trial. In addition, investigators will collect information about abnormal reactions monthly. All details of related and unexpected AEs, such as time of occurrence, degree of $\mathrm{AE}$, and suspected causes, will be recorded on CRFs. There is also a data safety monitoring board to oversee the trial.

\section{Study-specific visits and procedures}

The schedule for all study-related procedures for all evaluations is shown in Table 1. For each procedure, subjects are to be assessed by the same investigator or site personnel whenever possible. The timing of each visit is relative to randomization (day 1). Baseline measures include demographic characteristics, medical history, medications, measurement of vital signs (temperature, blood pressure, breathing and heart rates), complete blood count, routine urine test, stool test, liver and kidney function tests, 12-lead electrocardiogram, cardiac ultrasound, TCM syndrome, and QLQ and SF36 scores (details in the Additional file 2). All baseline measurements excluding the medical history will be repeated with all participants at visit 4 and visit 6 . The TCM syndrome, QLQ, and SF-36 data will be recorded at visit 3 and visit 5 . AEs and outcome measurements will be recorded from visit 3 to visit 6 .

\section{Randomization and blinding}

Participants are randomized in a 1:1 ratio using a computer-generated, site-stratified, block randomization schedule. The study capsule will be labeled with sequential randomization numbers, and each patient will be assigned the lowest number available at each participating 
center. All patients, care providers, and attending physicians will be blinded to treatment assignment until the study is completed. The duplicated blinding codes will be given to the main research institution and the manufacturer to keep, and the blinding codes cannot be broken during the trial.

\section{Date entry and quality control of data}

CRFs have been used for data entry, and data from all participating centers will be imported into the clinical data management system (http://www.xyedc.com/). To maintain the quality of the data, we will adopt valid measures to ensure information accuracy, integrity, and authenticity. First, computer logic checks will be run to identify items such as inconsistent dates, missing data, and questionable values. After that, the supervisor will perform source data verification to check the consistency of the original data. Queries may be issued by the supervisor and will be answered by the site investigators (see in the additional file 3). Second, manual checks will identify more complicated and less common errors. Third, the supervisor will conduct the site visit to compare the electronic database with the source documents. Identified errors will be solved to ensure the data quality. Fourth, the Data Coordination Center will be in charge of data validation.

\section{Sample size calculation}

The sample size was calculated on the basis of expected reduction in cardiovascular events (cardiac death, allcause mortality, reoccurrence of MI, and any form of revascularization). A previous study suggested that the incidence for all major clinical cardiovascular events combined at 1 year after interventional treatment of CHD is $12.8 \%$ [20]. Therefore, the hypothesis of this study is to reduce the incidence of cardiovascular events to $7 \%$ in the treatment group. Given a type I error rate of $\alpha=0.05$, a power of $80 \%$ (type II error rate of $\beta=0.2$ ), the sample size for one arm needs to be 447 , resulting in $n=2 \times 447=894$ patients. Considering a dropout rate of $20 \%$, a total of 1097 patients needs to be allocated to reach the required number of patients for the efficacy analysis. For convenience of randomization, we decided to recruit 1100 patients. The formula used to calculate the sample size is as follows:

$$
\begin{aligned}
& n=\frac{p_{1}\left(1-p_{1}\right)+p_{2}\left(1-p_{2}\right)}{\left(p_{1}-p_{2}\right)^{2}}\left(u_{\alpha}+{ }^{\alpha} u_{\beta}{ }^{\beta}\right)^{2} \\
& \mathrm{n} \approx 447 \text { patients/group }
\end{aligned}
$$

\section{Statistical analysis}

The data from all participating centers will be combined for statistical analysis of the primary and secondary endpoints as well as AEs. The analysis will be done at
Beijing Jiaotong University. Continuous variables will be presented as the mean $\pm \mathrm{SD}$. The comparability of the characteristics between the two study groups will be assessed using a two-samples Student's $t$ test for continuous variables and the $\chi^{2}$ test or Wilcoxon test, when appropriate, for categorical variables. The Wilcoxon paired signed-rank test will be used for within-group comparisons.

All randomized patients constituted the intention-totreat population. All participants will be analyzed according to their original treatment allocation. Primary and secondary endpoint data will be collected for the entire follow-up period for all patients. Patients lost to follow-up will be considered at risk until the date of last contact, at which point they will be censored. Kaplan-Meier curves will be used to examine MACE-free survival time. The Cox proportional hazards model with covariates of treatment will be used for primary and secondary analyses. Hazard ratios and $95 \%$ confidence intervals for each of the categories determined by age, sex, diabetes, and conventional therapy will be provided for the primary and secondary endpoints.

For all analyses, a value of $P<0.05$ will be considered statistically significant, and all tests will be two-tailed. All analyses will be conducted using SAS software version 9.2 (SAS Institute, Cary, NC, USA).

\section{Discussion}

In this trial, we will investigate whether the Xinyue capsule combined with conventional treatment reduces the incidence of MACE and improve quality of life among patients within 1 year after PCI. With the pleiotropic effects that encompass antioxidant, antiapoptosis, and improvement of insulin resistance, $\mathrm{PQS}$ was demonstrated to modify regional endothelial function, decrease oxidative stress and blood glucose, and induce angiogenesis [21-24]. In our previous studies, we have found that Xinyue capsules plus Chuanxiong capsules combined with conventional treatment can further reduce the incidence of cardiovascular events without any adverse effect in patients with ACS after PCI [16]. A small-sample clinical trial in which researchers recruited 100 patients with ACS after successful PCI showed that Xinyue capsules together with Western medicine treatment can improve cardiac function and quality of life [25]. These findings and observations serve as an impetus for large controlled trials to examine the effect of Xinyue capsules in patients with ACS after successful PCI. The present study is designed as a multicenter, double-blind, randomized, placebo-controlled, parallel-group superiority trial that will provide higherpowered evidence regarding long-term survival with the use of Xinyue capsules in addition to conventional treatment for patients with CHD after successful PCI. 
Our study has some limitations. First, the study is being undertaken in China, so it is uncertain whether the relative effects of the trial drugs would be similar in other ethnic groups. The relatively short follow-up period is another limitation of the study.

To conclude, the aim of this trial is to demonstrate that Xinyue capsules plus conventional treatment will lead to a reduction in the incidence of MACE in patients with CHD after successful PCI and subsequently yield long-term benefit.

\section{Trial status}

The trial was initiated in December 2014 and is currently open for enrollment. A total of 452 participants have been enrolled. However, no analysis has been conducted since the commencement of the trial.

\section{Additional files} Additional file 1: Institutional review board of 25 participating centers.
(PDF $82 \mathrm{~kb}$ )

Additional file 2: Case report form 2014.3.4R2. (PDF $1119 \mathrm{~kb}$ )

Additional file 3: Investigators brochure R2. (PDF $943 \mathrm{~kb}$ )

\section{Abbreviations}

ACS, acute coronary syndrome; $A E$, adverse event; $C H D$, coronary heart disease; CK, creatine kinase; CRF, case report form; ECG, electrocardiogram; hs-CRP, high-sensitivity C-reactive protein; MACE, major adverse cardiovascular event; Ml, myocardial infarction; $\mathrm{PCl}$, percutaneous coronary intervention; $\mathrm{PQS}$, Panax quinquefolius saponin; QLQ, Quality of Life Questionnaire; SF-36, 36-item Short Form Health Survey; TCM, traditional Chinese medicine; ULN, upper limit of normal

\section{Acknowledgements}

We thank Beijing Jiaotong University for their cooperation and technological support with the statistical analysis. We appreciate the help and efforts of all 25 research staff for participating in this trial and recruiting and treating the patients. This trial was supported financially by the National Basic Research Program (973 program) (number 2015CB554402) and the Jilin Province major science and technology achievements transformation project (number 11ZDZH005).

\section{Authors' contributions}

WPL, SDZ, ZYS, and WYH conceptualized and designed the study. GM drafted the manuscript, participated in data collection, and analyzed the data in collaboration with ZMJ, XRX, YQN, and BRN. WPL and SDZ reviewed the protocol for important intellectual content. All authors read and approved the final manuscript.

\section{Competing interests}

The authors declare they have no competing interests.

\section{Ethics approval and consent to participate}

The study is in compliance with the Declaration of Helsinki (Edinburgh 2000 version). The study protocol was reviewed and approved by the institutional review boards of 25 participating hospitals, and Additional file 1 shows this in more detail. Only clinicians holding the necessary qualifications are acting as principal investigators. Written informed consent will be obtained from each participant prior to enrollment.

\section{Author details}

${ }^{1}$ Cardiovascular Diseases Center, Xiyuan Hospital, China Academy of Chinese Medical Sciences, 1 Xiyuan Caochang, Haidian District, Beijing 100091, China. ${ }^{2}$ Department of Medicine, Jilin Jilin Yisheng Pharmaceutical Co., Ltd., 17 Wen Hua Dong Lu Road, Ji'an 134200, China.
Received: 16 March 2016 Accepted: 29 July 2016

Published online: 18 August 2016

\section{References}

1. Levine GN, Bates ER, Blankenship JC, Bailey SR, Bittl JA, Cercek B, et al. 2011 ACCF/AHA/SCAI Guideline for Percutaneous Coronary Intervention: a report of the American College of Cardiology Foundation/American Heart Association Task Force on Practice Guidelines and the Society for Cardiovascular Angiography and Interventions. J Am Coll Cardiol. 2011;58(24):e44-122.

2. Task Force on the Management of ST-Segment Elevation Acute Myocardial Infarction of the European Society of Cardiology (ESC). ESC guidelines for the management of acute myocardial infarction in patients presenting with ST-segment elevation. Eur Heart J. 2012;33(20):2569-619.

3. Sedlis SP, Hartigan PM, Teo KK, Maron DJ, Spertus JA, Mancini GB, et al. Effect of $\mathrm{PCl}$ on long-term survival in patients with stable ischemic heart disease. N Engl J Med. 2015;373(20):1937-46.

4. Task Force on the Management of Stable Coronary Artery Disease of the European Society of Cardiology. 2013 ESC guidelines on the management of stable coronary artery disease. Eur Heart J. 2013;34(38):2949-3003.

5. Hao PP, Jiang F, Chen YG, Yang J, Zhang K, Zhang MX, et al. Evidence for traditional Chinese medication to treat cardiovascular disease [published erratum appears in Eur Heart J. 2014;35(33):2260-1.]. Nat Rev Cardiol. 2015;12(6):374.

6. Wu SF, Zuo DY, Yu XF, Lv ZZ, Zhao XZ. Antimyocardial ischemic effects of Panax quinquefolium 20 s-protopanaxdiol saponins (PQDS) and its mechanism [in Chinese]. Chin Pharm J. 2002:02:100-3.

7. Ding $T, X u H B$, Sun $X B$, et al. Antimyocardial ischemic effect of Panax quiquefolius saponin [in Chinese]. Zhong Yao Yao Li Yu Lin Chuang. 2002;04:14-6.

8. Ma QY, Zhou W, Gao M, et al. Experimental research on protective effect of Panax quinquefolium saponin treatment against middle molecular substance induced cardiotoxicity in cultured myocardial cells [in Chinese]. Zhongguo Zhong Xi Yi Jie He Za Zhi. 2000;02:79-81.

9. Wang $\mathrm{CL}$, Shi DZ, Yin HJ, et al. Effect of Panax quinquefolius saponin on angiogenesis and expressions of VEGF and bFGF in myocardium of rats with acute myocardial infarction [in Chinese]. Zhongguo Zhong Xi Yi Jie He Za Zhi. 2007:04:331-4.

10. Wang $W$, Zhao XZ, Sui DY, et al. The effect of PQSs on experimental ventricular remodeling in rat [in Chinese]. Zhongguo Lao Nian Xue Xue Hui. 2008;18:1785-7.

11. LX G, Zhai FG, Nie $Y$, et al. Effect of Panax quinquefolium saponins from stem and leaves on neuronal apoptosis and the expression of caspase-3 in focal cerebral ischemia injury rats [in Chinese]. Zhong Yao Yao Li Yu Lin Chuang. 2008;01:30-2

12. Green DR, Kroemer G. Pharmacological manipulation of cell death: clinical applications in sight? J Clin Invest. 2005;115(10):2610-7.

13. Zhou MX, Xu H, Shi DZ, et al. The effect of Panax quinquefolius saponin on the gene expressions of perilipin and CD36 assosciated with lipid metabolism of apolipoprotein E-gene knockout mice [in Chinese]. Zhongguo Dong Mai Ying Hua Za Zhi. 2007;12:881-4.

14. Yin HJ, Zhang Y, Yang LH, et al. The effect of PQS on glucose transport, GLUT4 traslocation and CAP mRNA expression of adipocytes [in Chinese]. Zhongguo Yaolixue Tongbao. 2007;10:1332-7.

15. Zhang $Y$, Chen KJ, Yang LH. Effects of Panax quinquefolius saponin of stem and leaf on glucose-lipid metabolism and insulin signal transduction in insulin resistant model adipocytes [in Chinese]. Zhongguo Zhong Xi Yi Jie He Za Zhi. 2010;30(7):748-51.

16. Wang SL, Wang CL, Wang PL, Xu H, Liu HY, Du JP, et al. Combination of Chinese Herbal Medicines and Conventional Treatment versus Conventional Treatment Alone in Patients with Acute Coronary Syndrome after Percutaneous Coronary Intervention (5C Trial): an open-label randomized controlled, multicenter study. Evid Based Complement Alternat Med. 2013:2013:741518

17. Campbell MK, Piaggio G, Elbourne DR, Altman DG. CONSORT 2010 statement: extension to cluster randomised trials. BMJ. 2012;345, e5661.

18. Ke YN, Chen JL. Guideline for diagnosis and treatment of patients with unstable angina and non-ST-segment elevation myocardial infarction [in Chinese]. Zhonghua Xin Xue Guan Bing Za Zhi. 2007;04:295-304.

19. Standards of coronary heart disease for TCM syndrome differentiation [in Chinese]. Zhongguo Zhong Xi Yi Jie He Za Zhi. 1991;(05):257. 
20. Räber L, Kelbæk H, Ostojic M, Baumbach A, Heg D, Tüller D, et al. Effect of biolimus-eluting stents with biodegradable polymer vs bare-metal stents on cardiovascular events among patients with acute myocardial infarction: the COMFORTABLE AMI randomized trial. JAMA. 2012;308(8):777-87.

21. Wang B, Liu Y, Shang Q, Zhang Q, Zhang L, Liu J, et al. Interaction of Panax quinquefolius saponin and dual antiplatelets on vascular endothelial function in rats with acute myocardial infarction. Biomed Res Int. 2015;2015:932751.

22. Xie JT, Shao ZH, Vanden Hoek TL, Chang WT, Li J, Mehendale S, et al. Antioxidant effects of ginsenoside Re in cardiomyocytes. Eur J Pharmacol. 2006;532(3):201-7.

23. Vuksan V, Sievenpiper JL, Koo VY, Francis T, Beljan-Zdravkovic U, Xu Z, et al. American ginseng (Panax quinquefolius $L$ ) reduces postprandial glycemia in nondiabetic subjects and subjects with type 2 diabetes mellitus. Arch Intern Med. 2000;160(7):1009-13.

24. Hong SJ, Wan JB, Zhang Y, Hu G, Lin HC, Seto SW, et al. Angiogenic effect of saponin extract from Panax notoginseng on HUVECs in vitro and zebrafish in vivo. Phytother Res. 2009;23(5):677-86.

25. Liu HY, Wang CQ, Xie YS, Yin ZF, Bian L. Effect of Xinyue capsules on prognosis of patients with acute coronary syndrome after percutaneous coronary intervention [in Chinese]. Zhong Cheng Yao. 2015;06:1191-6.

\section{Submit your next manuscript to BioMed Central} and we will help you at every step:

- We accept pre-submission inquiries

- Our selector tool helps you to find the most relevant journal

- We provide round the clock customer support

- Convenient online submission

- Thorough peer review

- Inclusion in PubMed and all major indexing services

- Maximum visibility for your research

Submit your manuscript at www.biomedcentral.com/submit

) Biomed Central 